\title{
III. REPORTS
}

Bericht zum VII. Internationalen Forschungskolloquium „Junge Forschung im Bereich Deutsch als Fremdsprache“. Marburg \& weltweit 9.-10.07.2020

Das internationale Forschungskolloquium „Junge Forschung im Bereich Deutsch als Fremdsprache“ ist ein wichtiger Teil der gemeinsamen Kooperation der Arbeitsgruppe Deutsch als Fremdsprache des Instituts für Germanistische Sprachwissenschaft der Philipps-Universität Marburg, der Abteilung für Interkulturelle Glottopädagogik des Instituts für Angewandte Linguistik der Adam-Mickiewicz-Universität Poznań und der Abteilung Deutsche Sprache und Kultur / Niederlande-Deutschland-Studien der Radboud Universiteit in Nijmegen. Das Kolloquium findet einmal jährlich abwechselnd an einer der drei beteiligten Partner-Universitäten statt. Die bestehende grenzübergreifende Kooperation und die langjährige Fortführung des engen wechselseitigen Austauschs ist Prof. Dr. Sylwia Adamczak-Krysztofowicz, Dr. Sabine Jentges und Prof. Dr. Kathrin Siebold zu verdanken. Im Rahmen des Internationalen Forschungskolloquiums bekommen Nachwuchswissenschaftler und -wissenschaftlerinnen aus dem Bereich der Fremdsprachendidaktik und Sprachlehrforschung (Schwerpunkt: Deutsch als Fremdsprache) jedes Jahr die Möglichkeit, ihre laufenden Qualifikationsarbeiten (Masterarbeiten, Dissertationen, Habilitationen) vorzustellen und zu diskutieren. Ziel der zyklischen Veranstaltung ist eine qualitative Verbesserung von Qualifikations- und Forschungsarbeiten sowie die Anregung eines internationalen wissenschaftlichen Austauschs.

Die VII. Nachwuchskonferenz „Junge Forschung im Bereich Deutsch als Fremdsprache" fand am 9. und 10. Juli 2020 statt und wurde dank der Unterstützung der drei Partner-Universitäten und der Deutschen Gesellschaft für Fremdsprachenforschung (DGFF) erstmals digital ausgerichtet. Im virtuellen Meeting-Raum trafen sich am 9. und 10. Juli 2020 über fünfzig Teilnehmende aus der ganzen Welt. In jeweils 20-minütigen Referaten wurden zwanzig aktuell laufende Forschungsarbeiten von Teilnehmenden aus Deutschland, den Niederlanden, Polen, Japan, Neuseeland und Kamerun vorgestellt und anschließend diskutiert. Darunter vertreten waren Forschungszentren aus Nijmegen, Utrecht, Lüneburg, Leipzig, Duisburg-Essen, Marburg, Halle-Wittenberg, München, Bielefeld, Augsburg, Auckland, Kashiwa und Poznań.

Einführend referierte Patrick Wolf-Farré aus Duisburg-Essen, der in seinem Beitrag das Forschungsprojekt „Sprache, Herkunft und Identität in superdiversen Jugendgruppen " vorstellte. Radboud Universiteit in Nijmegen repräsentierten Henning Meredig mit dem Beitrag „Unterrichtskulturen an weiterführenden Schulen in Deutschland und den Niederlanden aus interdisziplinärer Perspektive“, Jana Hermann mit dem Referat zum Thema „Grenzüber- 
schreitender Schulaustausch aus Lehrerperspektive“ und Eva Knopp und Fleur de Mulder, die in ihrem Referat "Transfereffekte im bilingualen Rechtschreiberwerb" thematisierten. Auch die Themen der an der Philipps-Universität in Marburg laufenden Forschungsarbeiten im DaF/DaZ-Bereich waren breit gefächert: Neissen Zribi referierte zum Thema „Strategien und sprachliche Modifizierungen des Sprechakts der Aufforderung bei tunesischen DaF-Lernern“, Jennifer Müller stellte die Ergebnisse der „Bedarfsanalyse sprachlicher und fachlicher Herausforderungen beim Lesen von Geschichtstexten“ vor, Mingyue Gu fokussierte in ihrer Präsentation „Syntaktische Strukturen der gesprochenen Sprache und ihre Darstellung unter Jugendlichen in monologischen YouTube-Videos“. Quentin Glosemeyer stellte in seinem Beitrag „Skalierter Wortschatzerwerb nach GER-Niveau: Profile Deutsch als Basis?“ bereits erste Ergebnisse der im Rahmen seiner Arbeit durchgeführten Wortschatzabgleiche dar.

Im Beitrag von Sabine Ferber lag der Schwerpunkt auf der Darstellung von „Syntaxtraining als Alternative zu den Grammatik-Übungen in unterrichtstragendenden Lehrwerken“. Die dritte Universität aus der trilateralen Arbeitsgruppe - die Adam-Mickiewicz-Universität in Poznań - wurde von Katarzyna Bieniecka-Drzymała mit dem Vortrag zum Thema „Förderung und Entwicklung der Selbstreflexion bei Fremdsprachenlernenden im SprachlernCoaching" vertreten.

Doris Abitzsch (Utrecht) stellte in ihrer Präsentation „Fachdidaktische Entwicklungsforschung als Brückenbauer zwischen Ausbildung und Arbeitsfeld“ das Projekt dar, das im Rahmen des Comenius-Subventionsprogramms eingereicht wurde und dessen Ziel es ist, eine Brücke zwischen Studium und Arbeitsfeld zu schlagen. Die letzte Referentin des ersten Tages, Hannah Reckhaus (Halle-Wittenberg), skizzierte in dem Vortrag „Kompetenzorientiertes Prüfen als didaktische und methodische Perspektive im Feld von Deutsch als Zweitsprache“ ihr Dissertationsprojekt, das sich zum Ziel setzt, den Gesamtaufbau des Deutschtests für Zuwanderer differenziert zu untersuchen.

Den zweiten Tag der Tagung eröffnete der Vortrag „Mehrschriftlichkeit. Zur Wechselwirkung von Schriftsprachkompetenzen in L1 und L2 am Beispiel der deutschsprachigen Minderheit in Ostbelgien“ von Verena Rasp aus München, in dem das Dissertationsprojekt zum Einfluss der Kontaktkultur auf die schriftsprachlichen Kompetenzen in der Erstsprache (L1) Deutsch und der Zweitsprache (L2) Französisch vorgestellt wurde.

Im Rahmen des Kolloquiums wurden anschließend noch folgende Dissertationsprojekte vorgestellt und diskutiert: Julia Rietze aus Auckland präsentierte in ihrem Beitrag das Design ihrer Arbeit zum Thema „Emotionen, Identitäten und Investitionen in den Sprachbiographien von Herkunftssprecherinnen und -sprechern des Deutschen in Neuseeland. Olga Czyżak (Kashiwa) fokussierte die Gesichtswahrungsstrategien Lernender in Bezug auf fehlerhafte Äußerungen und die daraus resultierenden Interaktionen während der Gruppenarbeit. Sie stellte die Ergebnisse ihrer Arbeit dar, in der Präsentation mit dem Thema „'Ich habe meinen Fehler bemerkt, aber...' - Gesichtswahrungsstrategien in Gruppenarbeitsphasen am Beispiel der Thematisierung von Fehlern".

In dem Beitrag „Sprachaneignung mittels Fernsehen“ stellte Amra Havkić (Bielefeld) forschungsmethodologische Herangehensweisen vor, bei ihrer Untersuchung von Kindern und Jugendlichen aus Bosnien-Herzegowina, die sich Deutsch hauptsächlich ungesteuert durch das Medium Fernsehen angeeignet haben. Auch Jacques Abel Onya (Augsburg) befasst sich 
in seiner Dissertationsarbeit mit dem Thema des Deutscherwerbs. Das Arbeitsdesign wurde in dem Beitrag „Deutsch mit dem Radio in Kamerun lernen: Eine empirische Untersuchung mit Schülergruppen in der Stadt Jaunde“ präsentiert.

Charlotte Wendt (Lüneburg) präsentierte ihr Dissertationsprojekt, in Rahmen dessen erforscht wird, inwieweit digitale Schreibtools und -apps einen Mehrwert für den Unterricht mit sprachlich-heterogenen Schülergruppen darstellen können. Ludwig Lier (Augsburg) sprach $\mathrm{zu}$ „Blended-Learning-Szenarien für die Staatsexamensvorbereitung am Lehramtsstandort Augsburg“. Der Abschluss der Tagung wurde mit einem Vortrag von Natallia Baliuk aus Leipzig eingeleitet, in dem die Referentin die „Förderung digitaler Kompetenzen angehender DaF/DaZ-Lehrender in der universitären Professionalisierung“ thematisierte.

Der Schwerpunkt der an die jeweiligen Beiträge anschließenden Diskussionen lag darauf, Fragestellungen, Herangehensweisen, Erhebungs- und Auswertungsmethoden sowie Erklärungsansätze zu kommunizieren und zu reflektieren mit dem Ziel, den Teilnehmerinnen und Teilnehmern neue inhaltliche und methodische Impulse für die eigene Forschung zu geben.

Das Kolloquium zeigte, wie wichtig der grenzüberschreitende wissenschaftliche Austausch in Bezug auf laufende Forschungsprojekte im Bereich Deutsch als Fremdsprache ist. Die Veranstaltung war wieder ein voller Erfolg, der Prof. Dr. Kathrin Siebold, Dr. Sabine Jentges und Prof. Dr. Sylwia Adamczak-Krysztofowicz zu verdanken ist. Dank des großen Engagements der Veranstalterinnen ist es auch in Zeiten der Kontaktbeschränkungen gelungen, die wissenschaftliche Kooperation aufrecht zu erhalten und einem weltweiten Online-Publikum neue Impulse für die eigene Forschung zu geben.

Auch wenn das Online-Format der Konferenz eine sehr gute Alternative darstellte und vor allem eine nahezu unbeschränkte Möglichkeit für die weltweite Teilnahme ermöglichte, sprachen beim Abschluss der Konferenz und Ausblick auf 2021 viele Teilnehmerinnen und Teilnehmer die Hoffnung aus, nächstes Jahr wieder zum traditionellen Format, d. h. der Präsenzveranstaltung zurückkehren zu können. Den persönlichen Austausch zwischen den Teilnehmerinnen und Teilnehmern kann ein digitales Format nämlich nicht vollständig ersetzen.

Received: 05.10.2020; revised: 09.10.2020

\section{LUIZA CIEPIELEWSKA-KACZMAREK}

luizac@amu.edu.pl

Uniwersytet im. Adama Mickiewicza w Poznaniu

ORCID: 0000-0001-9255-493X

DOI: $10.14746 /$ gl.2020.47.2.12 
